\title{
Allogeneic CD123-specific Universal CAR123-expressing T-lymphocytes
}

National Cancer Institute

\section{Source}

National Cancer Institute. Allogeneic CD123-specific Universal CAR123-expressing Tlymphocytes. NCI Thesaurus. Code C146806.

Allogeneic, off-the-shelf, universal transcription activator-like effector nuclease (TALEN)eng ineered T-lymphocytes that have been genetically modified to express a chimeric antigen receptor (CAR) targ eting the tumor-associated antigen (TAA) human interleukin3 receptor alpha chain (IL3RA; cluster of differentiation 123; CD123), with potential immunomodulating and antineoplastic activities. Upon transfusion of allogeneic CD123specific universal CAR123-expressing T-lymphocytes (UCART 123), these cells target and bind to cancer cells expressing CD123. This induces selective toxicity in and causes lysis of CD123-expressing tumor cells. CD123 is normally expressed on committed blood progenitor cells in the bone marrow; its overexpression is associated with both increased leukemic cell proliferation and aggressiveness. Using TALEN technology, the UCART 123 cells no longer express the endogenous $\mathrm{T}$-cell receptor (TCR) thereby abrog ating the potential induction of graft-versus-host disease (GvHD) by the donor T-cells. 